\title{
Integrated spectral properties of 7 galactic open clusters ${ }^{\star}$
}

\author{
A.V. Ahumada ${ }^{1}$, J.J. Clariá ${ }^{1}$, E. Bica ${ }^{2}$, and A.E. Piatti ${ }^{3}$ \\ 1 Observatorio Astronómico, Universidad Nacional de Córdoba, Laprida 854, 5000 Córdoba, Argentina \\ 2 Instituto de Física, Universidade Federal do Rio Grande do Sul, Av. Bento Gonçalves, 9500, Caixa Postal 15051, CEP \\ 91501-970, Porto Alegre RS, Brazil \\ 3 Universidad de Concepción, Departamento de Física, Casilla 160-C, Concepción, Chile
}

Received June 23; accepted September 21, 1999

\begin{abstract}
This paper presents flux-calibrated integrated spectra in the range $3600-9000 \AA$ for 7 concentrated, relatively populous Galactic open clusters. We perform simultaneous estimates of age and foreground interstellar reddening by comparing the continuum distribution and line strengths of the cluster spectra with those of template cluster spectra with known parameters. For five clusters these two parameters have been determined for the first time (Ruprecht 144, BH 132, Pismis 21, Lyngå 11 and $\mathrm{BH} 217$ ), while the results here derived for the remaining two clusters (Hogg 15 and Melotte 105) show very good agreement with previous studies based mainly on colour-magnitude diagrams. We also provide metallicity estimates for six clusters from the equivalent widths of CaII triplet and $\mathrm{TiO}$ features. The present cluster sample improves the age resolution around solar metal content in the cluster spectral library for population synthesis. We compare the properties of the present sample with those of clusters in similar directions. Hogg 15 and Pismis 21 are among the most reddened clusters in sectors centered at $l=270^{\circ}$ and $l=0^{\circ}$, respectively. Besides, the present results would favour an important dissolution rate of star clusters in these zones.
\end{abstract}

Key words: techniques: spectroscopic — open clusters: general

Send offprint requests to: A.V. Ahumada

e-mail: andrea@oac.uncor.edu

* Based on observations made at Complejo Astronómico El Leoncito, which is operated under agreement between the Consejo Nacional de Investigaciones Científicas y Técnicas de la República Argentina and the National Universities of La Plata, Córdoba and San Juan, Argentina.

\section{Introduction}

The determination of Galactic open cluster parameters such as reddening, age and metallicity is fundamental to understand the structure and evolution of the Galactic disk. Old and intermediate-age open clusters are excellent probes of early disk evolution, while young open clusters provide information about current star formation processes and they are key objects to delineate Galactic structure. Open clusters located in the direction of the Galactic centre are particularly important to improve our knowledge of the chemical evolution of the inner Galactic disk (see, e.g., Friel \& Janes 1993; Piatti et al. 1995). Many of these clusters, however, have not been studied in detail yet because they are affected by high interstellar absorption and/or strong field star contamination.

Fundamental open cluster parameters have been mostly derived from colour-magnitude diagrams (CMDs) and/or photometric studies of individual giants (see, e.g., Janes et al. 1988; Batinelli \& Capuzzo-Dolcetta 1991 and references therein; Clariá \& Mermilliod 1992; Meynet et al. 1993; Friel 1995; Clariá et al. 1999). Sometimes, however, integrated spectra can provide valuable independent information about reddening, age and metallicity (see, e.g., Piatti et al. 1998a, 1998b, 1999).

The present work is part of a systematic spectroscopic survey of open and globular (or candidates) clusters mostly located toward the Galactic centre. CCD integrated spectra of small objects, from which it is very difficult to obtain individual information about stars unaffected by contamination, are useful not only to recognize the nature of these objects (Bica et al. 1995), but also to determine fundamental parameters of open and globular clusters (see, e.g., Santos \& Bica 1993; Piatti 1996; Bica et al. 1998).

In this paper we increase the sample of Galactic open clusters with integrated spectroscopy. Our aim is to derive reddening and age for a sample of 7 unknown or poorly known open clusters mostly located toward the 
Table 1. Cluster sample

\begin{tabular}{lcrc}
\hline Cluster & $l\left(^{\circ}\right)$ & $b\left(^{\circ}\right)$ & $D\left(^{\prime}\right)$ \\
\hline Ruprecht 144 & 20.70 & -1.25 & $4^{\prime}$ \\
Melotte 105 & 292.89 & -2.45 & $4^{\prime}$ \\
BH 132 & 300.27 & -1.33 & $3^{\prime}$ \\
Hogg 15 & 302.04 & -0.24 & $2^{\prime}$ \\
Pismis 21 & 320.35 & -1.80 & $2^{\prime}$ \\
Lyngå 11 & 338.18 & 0.47 & $4^{\prime}$ \\
BH 217 & 346.77 & -1.51 & $4^{\prime}$ \\
\hline
\end{tabular}

Galactic centre by means of flux-calibrated integrated spectra. In some cases we also provide information about cluster metallicity. In the next section we present the spectroscopic observations and measurements of equivalent widths for absorption features. The procedures employed to derive age, reddening and metallicity are explained in Sect. 3. Individual objects are discussed in Sect. 4. A comparison with other clusters located in similar directions is given in Sect. 5, and the final conclusions are presented in Sect. 6 .

\section{Spectroscopic data}

\subsection{Cluster sample and observations}

Concentrated open clusters with small angular diameter $(D)$ are certainly those most suitable to carry out integrated spectroscopic observations. This is so because the cluster as well as the surrounding background sky regions must extend along the whole slit. Besides, the angular diameter requirement results from the fact that the cluster integrated spectrum must reflect the synthesis of its stellar populations. Old or intermediate-age clusters with many red giants, for example, should reflect the presence of these stars in their integrated spectra. Similarly, integrated spectra of young clusters should present an enhanced blue continuum with comparatively strong Balmer lines.

In this study we have selected 7 compact $\left(D \leq 4^{\prime}\right)$, relatively populous Galactic open clusters to allow good star sampling in the integrated spectra. The choice of these clusters is due not only to their compact nature and relatively high surface brightness, but also to the scarcity or absence of previous studies about them. Galactic coordinates and angular diameters taken from Lyngå (1987) are listed in Table 1 for the cluster sample.

The observations were carried out with the $2.15 \mathrm{~m}$ telescope at the Complejo Astronómico El Leoncito (CASLEO, San Juan, Argentina) in two runs in May 1995 and July 1998, respectively. In both runs, we employed a CCD camera containing a Tektronics chip of $1024 \times 1024$ pixels attached to a REOSC spectrograph, the size of each pixel being $24 \times 24 \mu \mathrm{m}$. The slit was oriented in the east-west direction and the observations were made by scanning the slit across the objects in the north-south direction in order to get a proper sampling of cluster stars. The long slit, corresponding to $4.7^{\prime}$ on the sky, allowed us to sample regions of background sky. A grating of 300 line/mm in two different set-ups, namely "blue nights" and "red nights", was used. During the blue nights, the spectral coverage was $\approx 3500-7000 \AA$, with an average dispersion in the observed region of $\approx 140 \AA / \mathrm{mm}$ $(3.46 \AA /$ pix $)$. The slit width was 4.2 resulting in a resolution of $\approx 14 \AA$, as measured by the mean $F W H M$ of the comparison lines. During the red nights, we obtained spectra over the range $5800-9200 \AA$, with a similar dispersion $(\approx 3.36 \AA /$ pix $)$ and a final resolution of $\approx 17 \AA$ (same slit width). In order to eliminate the second-order contamination, an OG 550 filter was employed. Series of 15 minute exposures were taken for the objects. The standard stars LTT 4364, EG 274, and LTT7379 (Stone \& Balwin 1983) and HD 160233 (Gutiérrez-Moreno et al. 1988) were observed for flux calibrations. The hot standard HD 160233 was also used for the telluric absorption corrections in the near-infrared. Bias, dome and twilight sky and tungsten lamp flat fields were taken and employed in the reductions.

The logbook of observations is provided in Table 2, by Cols.: (1) cluster designation, (2) date of observation, (3) spectral range as explained above, (4) number of exposures and duration in seconds, (5) full width half maximum $(F W H M)$ in arcseconds, determined by the seeing during observation, and (6) total area of the cluster scanned in our observations.

Reductions were carried out with the IRAF package following standard procedures at the Observatorio Astronómico of the National University of Córdoba. We subtracted the bias and used flat-field frames - previously combined - to correct the frames for high and low spatial frequency variations. Background sky subtractions were then performed using pixel rows from the same frame after cleaning cosmic rays from the background sky regions. We controlled that no significant background sky residuals were present on the resulting spectra. The cluster spectra were extracted and wavelength-calibrated by fitting observed He-Ar comparison lamp spectra with template spectra. The rms errors involved in these calibrations are tipically $0.70 \AA$ ( 0.20 pixel $)$ for the blue nights and $0.35 \AA$ ( 0.10 pixel $)$ for the red ones. Extinction corrections and flux calibrations were then applied. Finally, we eliminated the telluric absorption in the near-IR following the precepts outlined in Bica \& Alloin (1987).

In Figs. 1-7 (bottom) we present the calibrated integrated spectra of the observed clusters normalized to $F_{\lambda}=$ 1 at $\lambda \approx 6000 \AA$. Indeed, the different slopes of the continuum energy distributions are the result of combined reddening and age effects. Although a certain amount of field star contamination may be expected mainly from stars 
Table 2. Journal of observations

\begin{tabular}{lccccc}
\hline Cluster & $\begin{array}{c}\text { Date } \\
(\mathrm{UT})\end{array}$ & $\begin{array}{c}\text { Spectral } \\
\text { range }\end{array}$ & $\begin{array}{c}\text { Exposure } \\
\text { Time }(\mathrm{secs})\end{array}$ & $\begin{array}{c}F W H M \\
\left({ }^{\prime \prime}\right)\end{array}$ & $\begin{array}{c}\text { AREA } \\
\left({ }^{\prime} \times{ }^{\prime}\right)\end{array}$ \\
\hline Ruprecht 144 & $7 / 20 / 98$ & blue & $6 \times 900$ & 1.5 & $3 \times 4$ \\
& $7 / 22 / 98$ & red & $2 \times 900$ & 2.9 & \\
Melotte 105 & $7 / 23 / 98$ & red & $2 \times 900$ & 1.6 & \\
& $5 / 25 / 95$ & blue & $3 \times 900$ & 2.0 & $3.5 \times 4$ \\
& $5 / 27 / 95$ & red & $3 \times 900$ & 2.1 & \\
BH 132 & $5 / 29 / 95$ & red & $2 \times 900$ & 2.2 & \\
& $7 / 18 / 98$ & blue & $4 \times 900$ & 1.6 & $2.5 \times 2.5$ \\
& $7 / 20 / 98$ & blue & $2 \times 900$ & 1.5 & \\
Hogg 15 & $7 / 24 / 98$ & red & $4 \times 900$ & 1.5 & \multirow{2}{*}{$1 \times 1.5$} \\
& $5 / 24 / 95$ & blue & $4 \times 900$ & 2.2 & \\
& $5 / 23 / 95$ & red & $4 \times 900$ & 2.0 & \\
Pismis 21 & $5 / 29 / 95$ & red & $2 \times 900$ & 2.0 & \multirow{2}{*}{$1 \times 2$} \\
& $7 / 18 / 98$ & blue & $4 \times 900$ & 1.9 & \\
Lyngå 11 & $7 / 21 / 98$ & blue & $2 \times 900$ & 1.2 & \\
& $7 / 22 / 98$ & red & $4 \times 900$ & 1.3 & $3 \times 4$ \\
BH 217 & $7 / 19 / 98$ & blue & $4 \times 900$ & 1.2 & \\
& $7 / 22 / 98$ & red & $4 \times 900$ & 1.3 & $3 \times 3.5$ \\
& $7 / 19 / 98$ & blue & $6 \times 900$ & 1.2 & 3.5 \\
& $7 / 24 / 98$ & red & $4 \times 900$ & 1.5 & \\
\hline
\end{tabular}

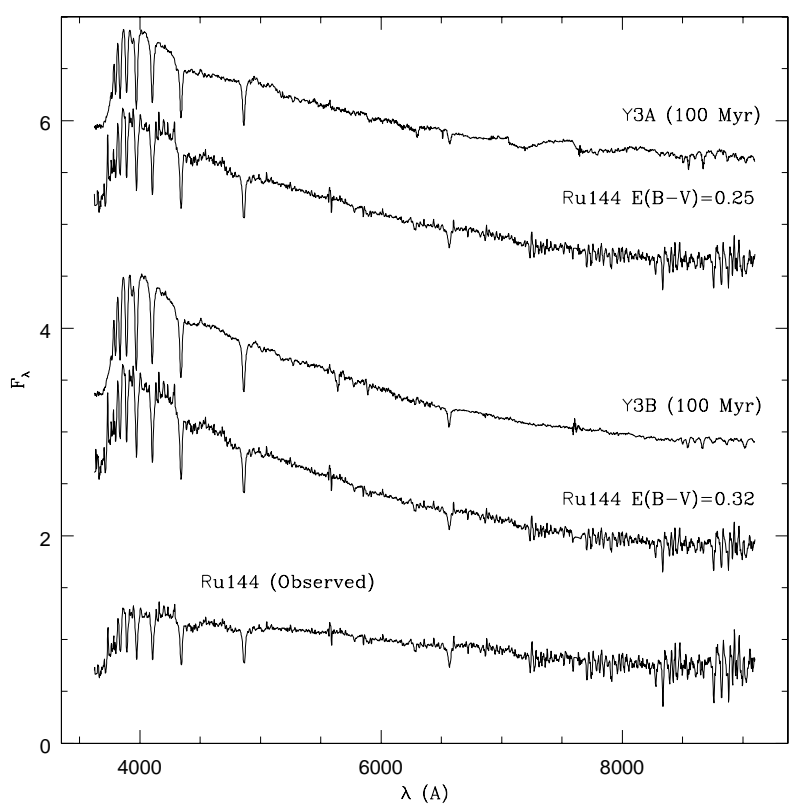

Fig. 1. Observed integrated spectrum of Ruprecht 144 (bottom), the spectrum corrected for the derived reddening $E(B-$ $V)=0.32$ and the Y3B template which best matches the spectrum. An alternative match using the Y3A template (top) is also shown. Spectra are in relative $F_{\lambda}$ units normalized at $\approx 6000 \AA$. Constants have been added to the spectra, except for the bottom one

located close to the cluster centre, only bright field stars could affect the spectra significantly but this is not the case in any of the observed clusters.

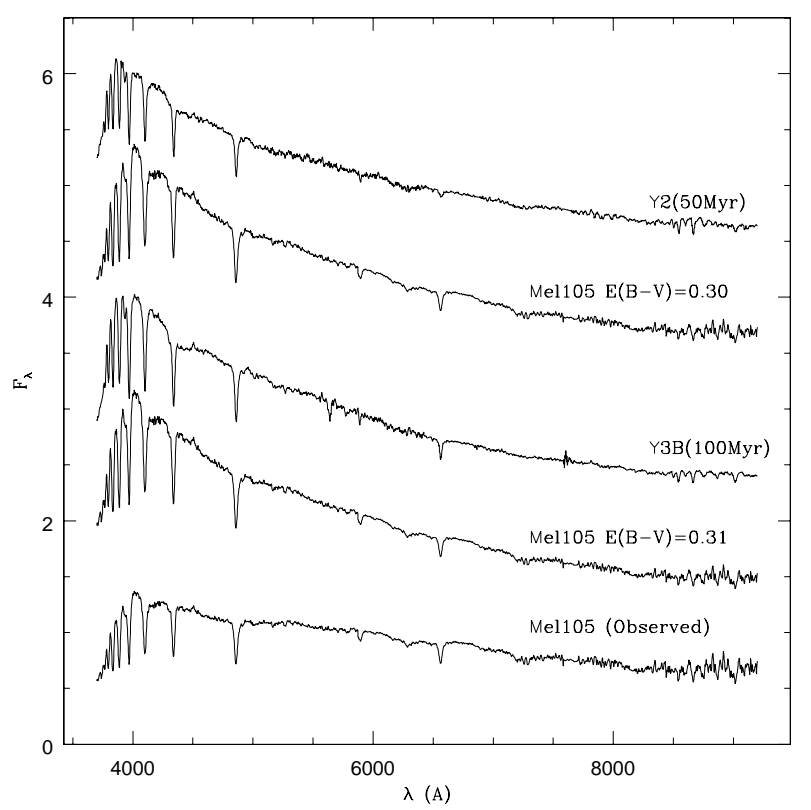

Fig. 2. Comparison of observed (bottom) and reddeningcorrected spectra of Melotte 105 with the Y3B and Y2 templates. Units as in Fig. 1

\subsection{Equivalent width measurements}

Equivalent widths (EWs) in some selected spectral windows defined by Bica \& Alloin (1986a, 1986b, 1987, hereafter BA86a, BA86b, and BA87, respectively) were measured for the cluster sample using the spectral analysis program SPEED (Schmidt 1988). The EWs of the Balmer lines were measured taking into account both the spectral windows and flux points as defined by BA86a, BA86b and BA87. Two alternative fits using high 


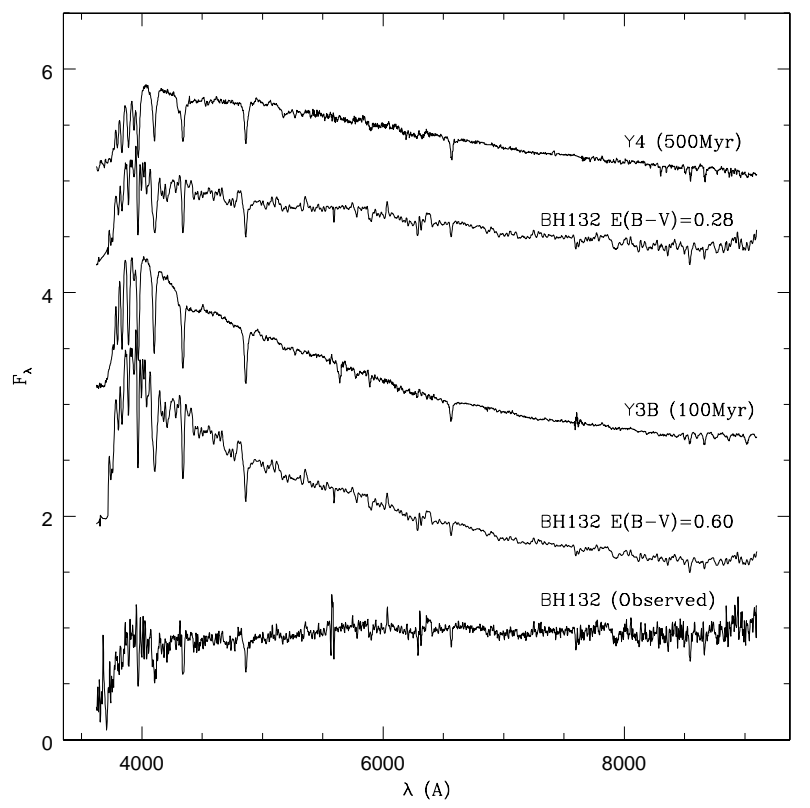

Fig. 3. Comparison of the observed (bottom) and reddeningcorrected spectra of $\mathrm{BH} 132$ with the Y3B and Y4 templates. Units as in Fig. 1

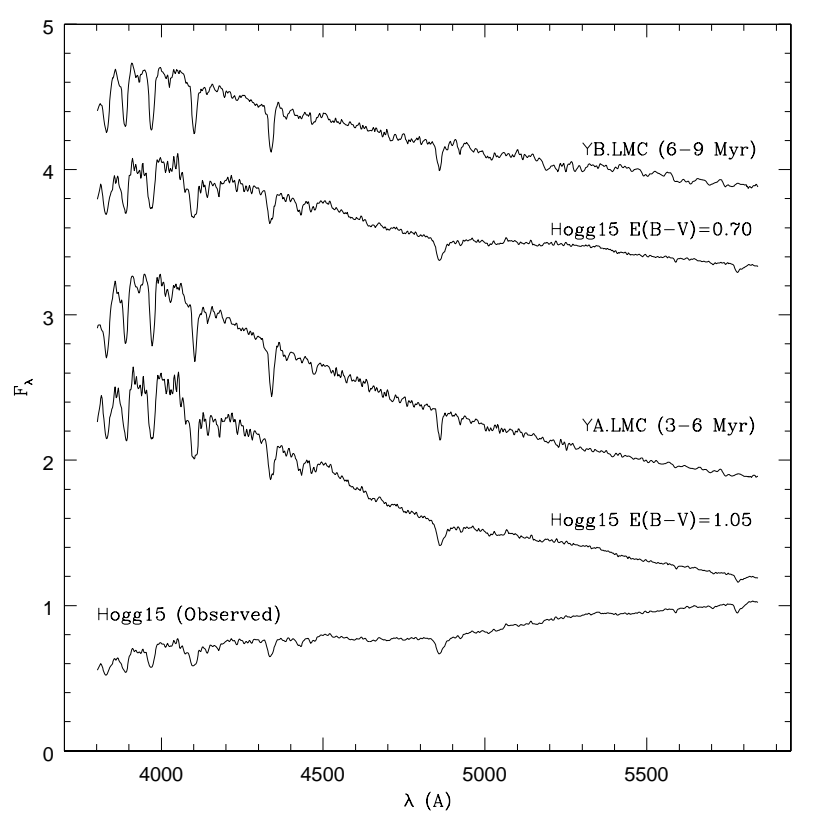

Fig. 4. Comparison of the observed (bottom) and reddeningcorrected spectra of Hogg 15 with the YA.LMC and YB.LMC templates. Units as in Fig. 1

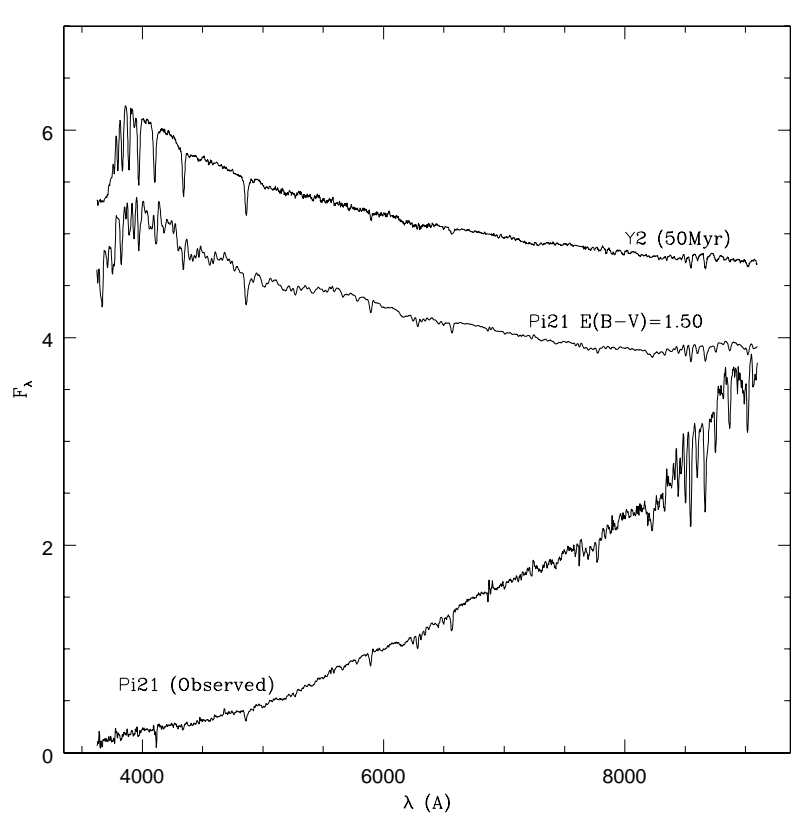

Fig. 5. Comparison of the observed (bottom) and reddeningcorrected spectra of Pismis 21 with the Y2 template which best matches the spectrum. Units as in Fig. 1

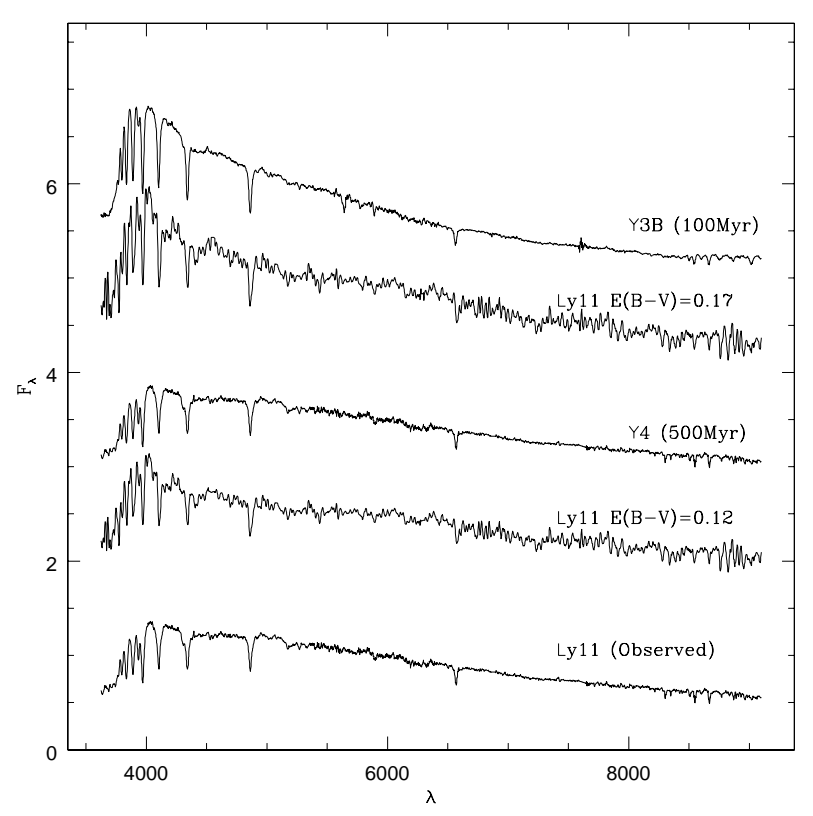

Fig. 6. Comparison of the observed (bottom) and reddeningcorrected spectra of Lyngå 11 with the Y4 and Y3B templates. Units as in Fig. 1 


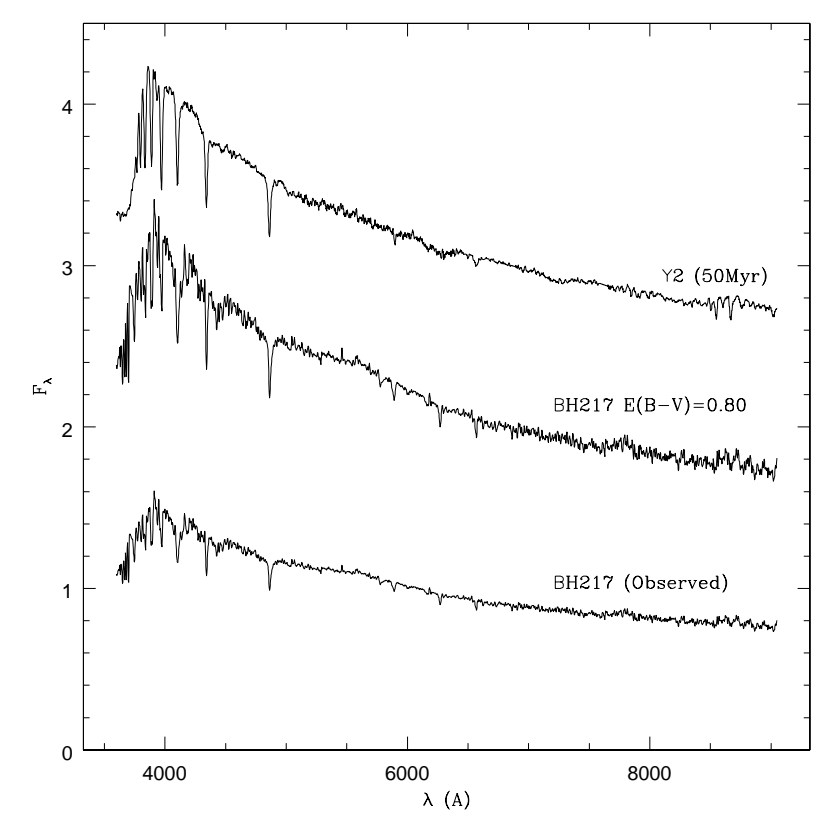

Fig. 7. Comparison of the observed (bottom) and reddeningcorrected spectra of $\mathrm{BH} 217$ with the Y2 template. Units as in Fig. 1

and low continuum tracings were made in order to take into account the spectral noise in the measurements of the Balmer-line EWs. The resulting uncertainties in the EWs are found to be smaller than those associated with the intrinsic dispersions in Table 2 of BA86b. Equivalent widths for $\mathrm{TiO}$ features between $(8200-8500) \AA$ and CaII triplet lines were also measured in six of the seven clusters. The results of the measurements are presented in Tables 3 and 4, where the EWs are given in Angström units $(\AA)$.

\section{Determination of fundamental cluster parameters}

BA86a and BA87 analyzed the behaviour of metallic and Balmer-line equivalent widths as well as the continuum energy distribution in the spectral range $3700-9700 \AA$ from integrated spectra of Galactic and Magellanic Cloud star clusters. Shortly afterwards, Bica (1988) created a library of template cluster spectra with known parameters grouped according to their evolutionary stages. The construction of spectral libraries of star clusters of all ages is a fundamental reference in order to study clusters in distant galaxies. Efforts in this direction have been made in different spectral ranges (e.g. Bica et al. 1990; Bonatto et al. 1995; Santos et al. 1995).

In this study, we have simultaneously estimated age and foreground interstellar reddening by comparing the continuum (and lines) of the observed cluster spectra with those of template cluster spectra. This procedure has been described by Santos \& Bica (1993). A direct reddeningindependent age estimate was first obtained from EWs of the Balmer lines in absorption in each spectrum by interpolating these values in the age calibration of BA86b (their Table II). Notice that the Balmer lines peak at $t$ (age) $\approx 500 \mathrm{Myr}$, but spectra of intermediate-age clusters have strong metallic lines in the blue range like $\mathrm{K}$ CaII. This is not the case of the present sample which ensures that we are dealing with ages younger than about $500 \mathrm{Myr}$. We then selected an appropriate set of template spectra according to the age provided by the Balmer lines and varied reddening and template to get the best match of continuum and lines of the observed spectrum to that of the template that most resembles it. To perform reddening corrections, we used the normal reddening law $A_{\lambda}=0.65$ $A_{v}(1 / \lambda-0.35)$ (Seaton 1979), the relation $A_{v}=3 \mathrm{E}(B-V)$, and the SPEED spectral analysis software (Schmidt 1988). We have adopted the above reddening law to keep internal consistency with the database of template spectra established using this law and the factor 3 for the ratio of total to selective absorption (e.g., Bica 1988; Santos et al. 1995). The resulting reddenings and ages for the cluster sample are shown in Table 5. We note that the uncertainty in the adopted reddening represents the lowest reddening variation necessary to distinguish the cluster spectrum from that of the corresponding template. As shown in Table 5, the uncertainties in the $E(B-V)$ determinations range from 0.02 to 0.05 magnitudes.

The template groups from Bica (1988) and Santos et al. (1995) found to be particularly useful in the present study are YA.LMC (age $\approx 3-6 \mathrm{Myr}$ ), YB.LMC (6 - 9 Myr), Y2 (50 Myr), Y3A and Y3B (100 Myr) and Y4 (500 Myr). The group Y3A has an enhanced population of massive asymptotic giant branch stars with respect to $\mathrm{Y} 3 \mathrm{~B}$ (BA87). The template sequence depends essentially on age, the metallicity effects being negligible in the blue-visual ranges.

As discussed by BA87, CaII triplet and other absorption windows in the near-IR can provide metallicity estimates in young clusters. The metallicity values interpolated from Table A3 of BA87, however, should be taken as indicative and, for definitive results for each cluster, complementary information from deep CMDs and/or spectroscopy of individual member giants would be necessary. Notice that for lower surface brightness clusters (e.g., Lyngå 11, Fig. 6), residuals of telluric line corrections may be important. Nevertheless, the CaII triplet region is little affected by such problems (BA87), which allows one to confidently measure them in such cases.

\section{Discussion on individual clusters}

All the clusters studied in this work have been classified as belonging to types I or II of Ruprecht (1966). There exist previous results only about Hogg 15 and Melotte 105, 
Table 3. Measurements of equivalent widths of four Balmer lines

\begin{tabular}{lrrrc}
\hline Cluster & $\mathrm{H}_{\delta}$ & $\mathrm{H}_{\gamma}$ & $\mathrm{H}_{\beta}$ & $\mathrm{H}_{\alpha}$ \\
Windows & $(4082-4124) \AA$ & $(4318-4364) \AA$ & $(4846-4884) \AA$ & $(6540-6586) \AA$ \\
\hline Ruprecht 144 & $9.0 \pm 0.1$ & $10.2 \pm 0.4$ & $8.4 \pm 0.2$ & $6.0 \pm 0.2$ \\
Melotte 105 & $11.4 \pm 0.2$ & $9.5 \pm 0.3$ & $10.2 \pm 0.4$ & $7.1 \pm 0.4$ \\
BH 132 & $15.6 \pm 0.4$ & $9.4 \pm 0.1$ & $7.2 \pm 0.2$ & - \\
Hogg 15 & $7.1 \pm 0.8$ & $4.0 \pm 0.7$ & $6.6 \pm 0.7$ & $1.2 \pm 0.1$ \\
Pismis 21 & $11.2 \pm 0.2$ & $7.5 \pm 0.1$ & $7.4 \pm 0.1$ & - \\
Lyngå 11 & $11.6 \pm 0.1$ & $9.0 \pm 0.1$ & $10.3 \pm 0.1$ & - \\
BH 217 & $9.2 \pm 0.2$ & $6.2 \pm 0.1$ & $7.2 \pm 0.1$ & $3.2 \pm 0.1$ \\
\hline
\end{tabular}

Table 4. Measurements of equivalent widths of $\mathrm{TiO}$ and CaII triplet features

\begin{tabular}{lcccc}
\hline $\begin{array}{l}\text { Cluster } \\
\text { Window }\end{array}$ & $\begin{array}{c}\text { TiO } \\
(8234-8476) \AA\end{array}$ & $\begin{array}{c}\text { CaII } \\
(8476-8520) \AA\end{array}$ & $\begin{array}{c}\text { CaII } \\
(8520-8564) \AA\end{array}$ & $\begin{array}{c}\text { CaII } \\
(8640-8700) \AA\end{array}$ \\
\hline Ruprecht 144 & - & $4 \pm 1$ & $5 \pm 2$ & $5 \pm 1$ \\
Melotte 105 & - & $4 \pm 1$ & $5 \pm 1$ & $5.3 \pm 0.8$ \\
BH 132 & $11 \pm 2$ & $4 \pm 2$ & $4 \pm 1$ & $5 \pm 1$ \\
Hogg 15 & $13 \pm 2$ & $3.2 \pm 0.7$ & - & $4.6 \pm 0.7$ \\
Pismis 21 & $13 \pm 1$ & $4 \pm 2$ & $5 \pm 1$ & $5 \pm 1$ \\
Lyngå 11 & $15 \pm 2$ & - & $5 \pm 1$ & $5 \pm 1$ \\
\hline
\end{tabular}

Table 5. Reddening and age determinations

\begin{tabular}{lcccc}
\hline Cluster & $E(B-V)$ & $\begin{array}{c}\text { Age (Balmer) } \\
(\mathrm{Myr})\end{array}$ & $\begin{array}{c}\text { Age (template match) } \\
(\mathrm{Myr})\end{array}$ & $\begin{array}{c}\text { Adopted age } \\
(\mathrm{Myr})\end{array}$ \\
\hline Ruprecht 144 & $0.32 \pm 0.02$ & 200 & 100 & $150 \pm 50$ \\
Melotte 105 & $0.31 \pm 0.02$ & 300 & 100 & $200 \pm 100$ \\
BH 132 & $0.60 \pm 0.05$ & 200 & 100 & $150 \pm 50$ \\
Hogg 15 & $1.05 \pm 0.05$ & 30 & $3-6$ & $5 \pm 2$ \\
Pismis 21 & $1.50 \pm 0.03$ & 110 & 50 & $80 \pm 30$ \\
Lyngå 11 & $0.12 \pm 0.03$ & 400 & 500 & $450 \pm 50$ \\
BH 217 & $0.80 \pm 0.03$ & 20 & 50 & $35 \pm 15$ \\
\hline
\end{tabular}

${ }^{a}$ We adopted the template method age, since for very young ages the Balmer lines lose sensitivity (BA86a).

which can be compared with the present ones. Precisely, the latter cluster was selected not only because of its compactness but also because it was observed by Santos \& Bica (1993) at La Silla (Chile) using the same spectroscopic technique, so that it can be used as control cluster. The five remaining clusters BH 132, BH 217, Lyngå 11, Pismis 21 and Ruprecht 144 have not been previously studied and their reddenings and ages have been determined here for the first time. Lyngå 11, Pismis 21 and Ruprecht 144 are mentioned in early catalogation or morphological classification works (Pismis 1959; Lyngå 1965; Ruprecht 1966).

\subsection{Ruprecht 144}

The integrated spectrum of Ruprecht 144, corrected for $E(B-V)=0.25$, is compared to that of the Y3A template (100 Myr) in Fig. 1. Notice that this template presents $\mathrm{TiO}$ bands not observed in Ruprecht 144 . Notice also that at $\approx 100 \mathrm{Myr}$ very populous LMC clusters may present strong TiO bands. This phenomenon appears to be as- sociated to AGB stars (BA87, Bica et al. 1990). Owing to statistical effects in non-massive clusters, the effect is not expected in Galactic open clusters. Indeed, the best match is found for the Y3B template (100 Myr) adopting $E(B-V)=0.32 \pm 0.02$ (Fig. 1). The age derived from the Balmer lines is slightly larger (Table 5). CaII triplet EWs yield $\left[Z / Z_{\odot}\right]=-0.1 \pm 0.2$.

\subsection{Melotte 105}

We have simultaneously determined the age and reddening for Melotte 105, also known as Collinder 246 (Collinder 1931), using the template Y3B (100 Myr). The best template match yields $E(B-V)=0.31 \pm 0.02$. Figure 2 shows the observed cluster spectrum, the spectrum corrected for the derived interstellar reddening $E(B-V)=$ 0.31 , and the template Y3B which best matches the cluster spectrum. An alternative match using the template Y2 (50 Myr) is also shown. The derived metallicity from CaII triplet EWs is $\left[Z / Z_{\odot}\right]=0.0 \pm 0.2$. The age and reddening here derived are in excellent agreement with 
the values found by Santos \& Bica (1993) using the same technique. On the other hand, using $U B V$ photoelectric photometry Sher $(1965)$ estimated the same age $(\approx 100$ Myr) and a somewhat larger mean reddening $E(B-V)=$ 0.38 . The non-uniform reddening determined photoelectrically by Sher (1965) may be reflecting the existence of internal dust associated to the cluster. If this is the case, the spectroscopic reddening should be lower than the photometric one since the less reddened stars of a given spectral type should contribute to the integrated light with larger fluxes in comparison with the most reddened stars of the same spectral type. In other words, as stated by Santos \& Bica (1993), it appears highly probable that the lower reddening derived from the integrated spectrum indicates an internal reddening, in the present case, of $\approx 0.07 \mathrm{mag}$.

Based on CCD $U B V$ data Kjeldsen \& Frandsen (1991) derived an age of $\approx 150 \mathrm{Myr}$ and a quite larger reddening $E(B-V)=0.52$. The difference found in the cluster reddening may be explained by the fact that Kjeldsen \& Frandsen $(B-V)$ colours are shifted by approximately $0.1 \mathrm{mag}$ with respect to those of Sher (1965). Finally, Balona \& Laney (1995) obtained $E(b-y)=0.32$ from uvby photometry, equivalent to $E(B-V)=0.43$, if the relation $E(B-V)=1.35 E(b-y)$ derived by Crawford (1978) is used. This reddening differs scarcely by 0.05 mag from that of Sher (1965), while the age estimated by Balona \& Laney (250 Myr) is somewhat larger than the present one.

\section{3. $B H 132$}

BH 132 was first recognized as an open cluster by van den Bergh \& Hagen (1975). Figure 3 shows the integrated cluster spectrum, corrected for $E(B-V)=0.28$, compared to the Y4 template (500 Myr). Note that although a general resemblance between these two spectra is apparent, neither the Balmer jump nor the $\mathrm{H} \alpha$ line show a good agreement. Besides, the CaII K line in BH 132 appears to be more diluted, which cannot be a metallicity effect since CaII triplet near $8600 \AA$ appears to be similar in both the template and BH 132 spectra. This difference would be explained if BH 132 were younger than the Y4 template. The best match is found for the Y3B template (100 Myr), using $E(B-V)=0.60$ (Fig. 3). Note that the spectral properties of this cluster are very similar to those of Ruprecht 144 and Melotte 105. Equivalent widths of TiO and CaII triplet features (Table 4) are compatible with the cluster having a nearly solar metal content.

\section{4. $\operatorname{Hogg} 15$}

Hogg 15, also known as BH 139 (van den Bergh \& Hagen 1975) or ESO95SC15 (Lauberts 1982), was first recognized as an open cluster by Hogg (1965a, 1965b). Photoelectric

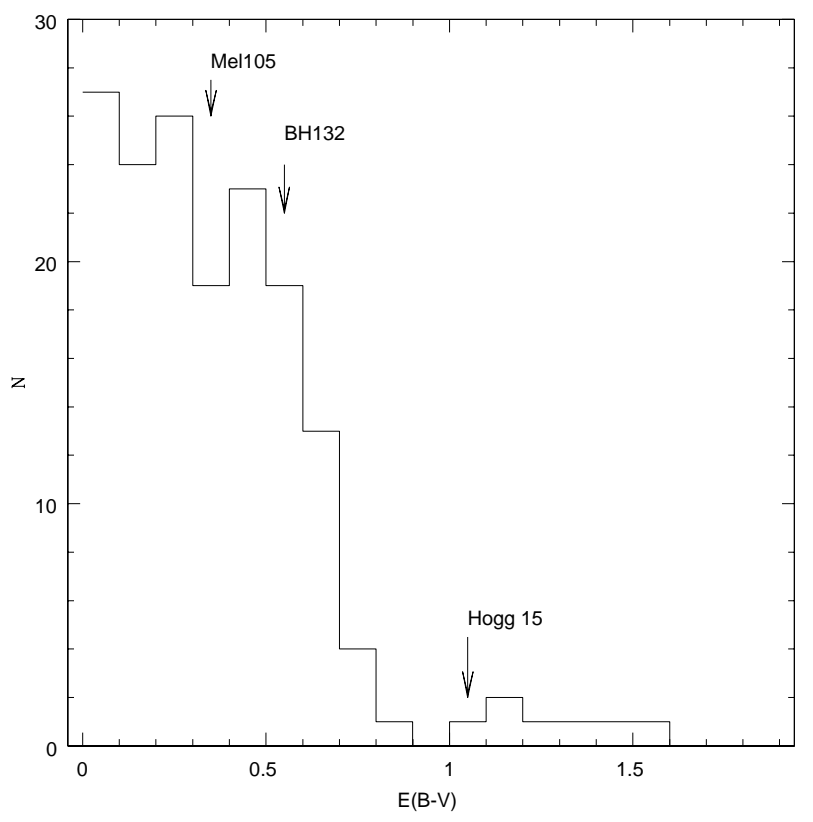

Fig. 8. Reddening histogram in the Galactic sector centered at $l=270^{\circ}$

$U B V$ photometry by Moffat (1974) yields $E(B-V)=$ $1.16 \pm 0.03$, a distance of $4.2 \mathrm{kpc}$, and an age $\leq 8 \mathrm{Myr}$. Therefore, Hogg 15 is one of the few clusters known to lie in the inner arm -II, much further behind the Coalsack than the adjacent cluster NGC 4609, which is located at about $1.3 \mathrm{kpc}$ from the Sun and is reddened by $E(B-V)$ $=0.36$ (Feinstein \& Marraco 1971). The above reddening and age of Hogg 15 are in very good agreement with the results obtained from integrated spectroscopy. We show in Fig. 4 two template matches. The template match with YB.LMC $(6-9 \mathrm{Myr})$ gives $E(B-V)=0.70 \pm 0.05$, whereas YA.LMC $(3-6 \mathrm{Myr})$ yields $E(B-V)=1.05 \pm$ 0.05. The latter is clearly the best match so that Hogg 15 is confirmed as a highly reddened young open cluster. We point out that we also dispose of the near-IR spectrum for Hogg 15, but we limit our comparison in Fig. 4 to the range in common with the available template. The derived metallicity is $\left[Z / Z_{\odot}\right]=-0.2 \pm 0.2$. Moffat (1974) and Smith et al. (1994) have discussed the possibility that the WN 6 star HDE 311884 could be a cluster member. The cluster age is clearly compatible with such possibility (cf. Pacziński 1973).

\subsection{Pismis 21}

Pismis 21 was first recognized as an open cluster by Pismis (1959). Notice the extreme absorption effects in the observed spectrum (Fig. 5). We also show the foreground reddening corrected spectrum for $E(B-V)$ $=1.50 \pm 0.03$ and the Y2 template $(50 \mathrm{Myr})$ which best matches the spectrum. Although the age from Balmer 


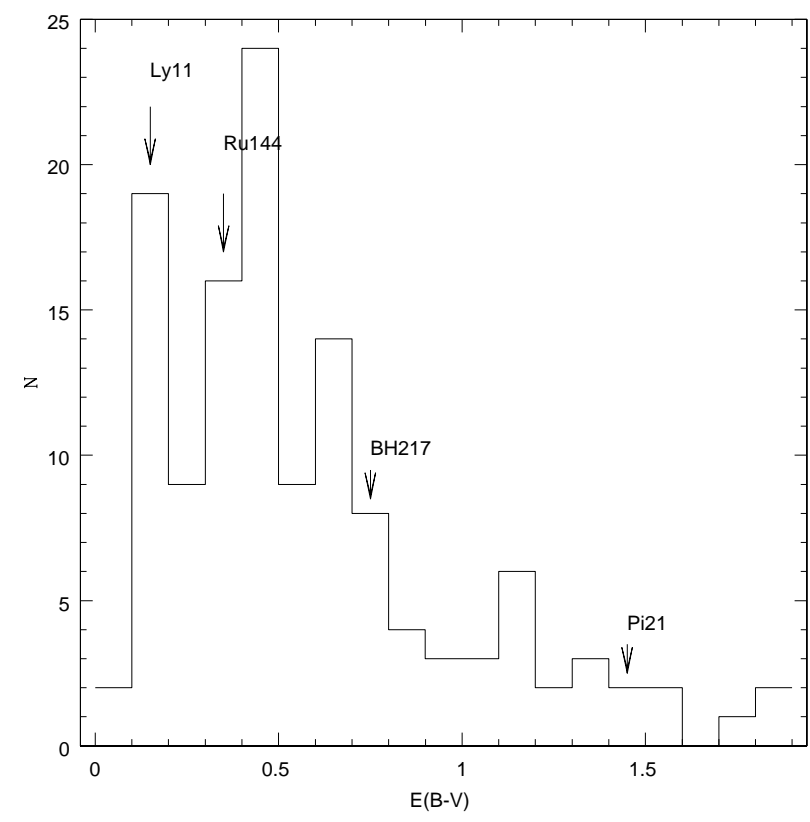

Fig. 9. Same as Fig. 8 for $l=0^{\circ}$

lines is slightly greater (Table 5), Pismis 21 is found to be a moderately young highly reddened open cluster. The derived metallicity is $\left[Z / Z_{\odot}\right]=0.0 \pm 0.2$.

\subsection{Lyngå 11}

We show in Fig. 6 two template matches. The template match with Y3B $(100 \mathrm{Myr})$ gives $E(B-V)=0.17 \pm 0.03$, whereas Y4 (500 Myr) yields $E(B-V)=0.12 \pm 0.03$. An inspection of the spectra reveals that the incipient $4000-\AA$ break favours Y4 as a match, as well as the general spectral distribution. More details on the Balmer jump and 4000- $\AA$ break as a function of age are given in Bica et al. (1994). Since the Balmer-line method also gives an age closer to Y4 (Table 5), we adopt the latter match as the best solution. The derived metallicity from CaII triplet and $\mathrm{TiO}$ features is $\left[Z / Z_{\odot}\right]=+0.1 \pm 0.2$.

\section{7. $B H 217$}

This cluster was first recognized by van den Bergh \& Hagen (1975), and is also known as ESO333SC2 (Lauberts 1982). The solution for BH 217 yields an age of about $50 \mathrm{Myr}$ and $E(B-V)=$

$0.80 \pm 0.03$ by template match (Fig. 7). The age derived from the Balmer lines is only slightly lower (Table 5) and no metallicity could be derived for this cluster. The spectral features of $\mathrm{BH} 217$ are quite similar to those of the more reddened cluster Pismis 21 (Fig. 5).

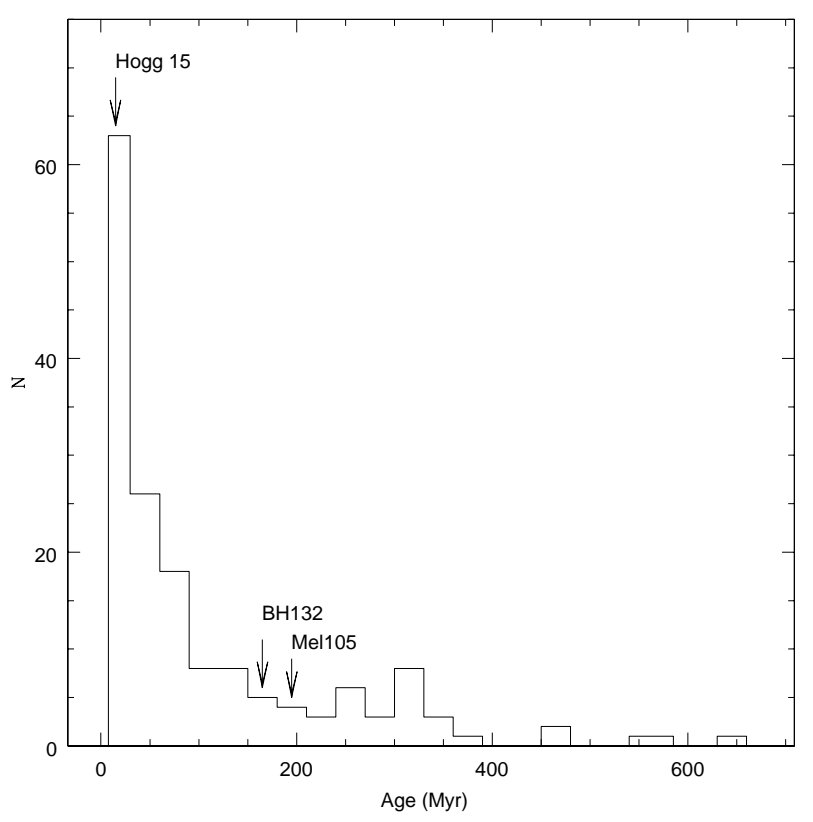

Fig. 10. Age histogram in the Galactic sector centered at $l=$ $270^{\circ}$

\section{Comparison with other clusters in similar directions}

The present sample of 7 clusters belongs to the young disk. Although small, it is interesting to compare it with open clusters already studied in the literature in similar disk zones. This is because the present sample deals with relatively faint clusters, on the average fainter than more prominent objects which have had CMD studies in the literature. We must recall that clusters like the present ones are numerous in catalogs and will surely be the most frequent kind of young disk targets for CMD studies in coming years.

The WEBDA database (Mermilliod 1999) keeps updated parameters from studies in the literature. We selected all young disk clusters (about 600 Myr or younger, we recall that Hyades age clusters or older characterizing the old disk have their own properties and observational requirements) in $90^{\circ}$ sectors centered respectively at $l=270^{\circ}$ and towards the Galactic center $\left(l=0^{\circ}\right)$. There are respectively 163 and 130 clusters, while our sample includes respectively 3 and 4 objects in such zones.

We show in Figs. 8 and 9 the reddening histograms in the two sectors respectively, and in Figs. 10 and 11 the age histograms respectively. The properties of the present clusters are indicated by arrows. Reddening and especially age distributions in both sectors are similar. Although relatively faint, only Hogg 15 and Pismis 21 are highly reddened in our sample, and they are also among the most reddened in the sectors. The frequency decrease of clusters with increasing age is remarkable. The distribution must reflect the formation/dissolution rates of star 


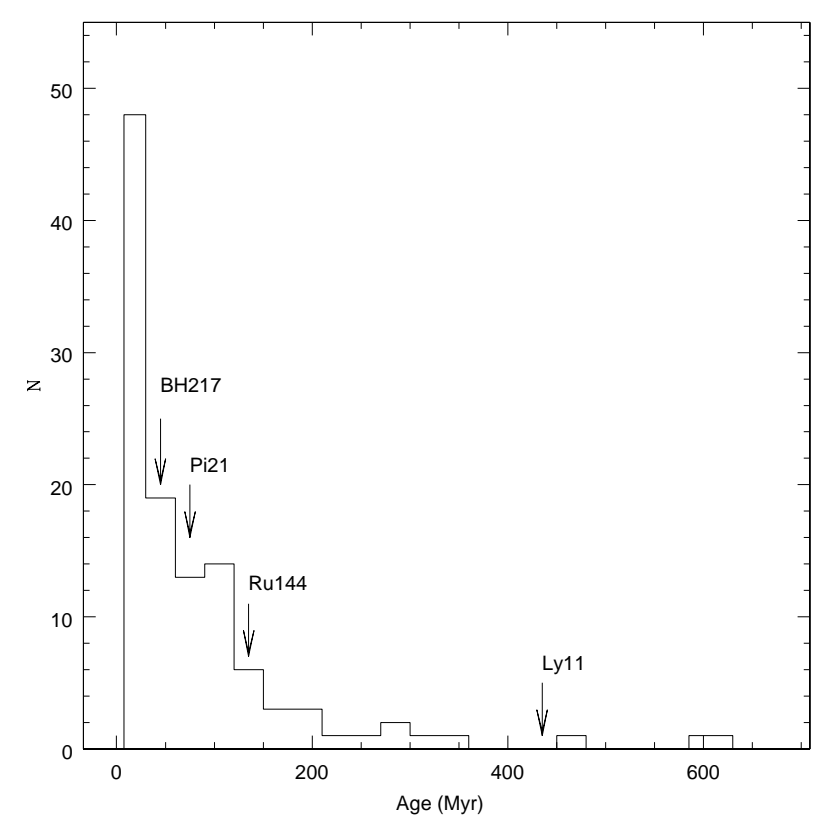

Fig. 11. Same as Fig. 10 for $l=0^{\circ}$

clusters (Wielen 1971, 1988), perhaps biased by observational constraints. However, what our small faint sample suggests is that the distribution will not change much in the future when new faint clusters are observed. Only one cluster (Lyngå 11) occurs for larger ages. Since an observational bias appears improbable, the age histograms must intrinsically indicate a real drop. Unless major star forming events had occurred in the Galactic disk in the last $100 \mathrm{Myr}$ or so, the results would favour an important dissolution rate of star clusters.

\section{Conclusions}

In the present paper we have studied a sample of 7 Galactic open clusters by means of integrated spectroscopy in the range $3600-9000 \AA$. We have mainly estimated ages and foreground reddenings from the comparison of the cluster integrated spectra with template cluster spectra. Hogg 15 is found to be a very young open cluster (age $\approx 3-6 \mathrm{Myr}$ ), while BH 132, BH 217, Pismis 21, Melotte 105 and Ruprecht 144 are moderately young (35 - 200 Myr). Lyngå 11 (450 Myr) is the oldest cluster in the present sample. The derived reddenings range from $E(B-V)=0.12$ in Lyngå 11 to $E(B-V)=1.50$ in Pismis 21. The results here derived for Hogg 15 and Melotte 105 show very good agreement with previous studies based mainly on CMDs. The present sample improves the age resolution around solar metallicity in the cluster spectral library for population synthesis. We have compared the properties of the present cluster sample with those of open clusters younger than
$600 \mathrm{Myr}$, already studied in the literature in similar disk zones. Hogg 15 and Pismis 21 appear to be among the most reddened clusters in sectors centered at $l=270^{\circ}$ and $l=0^{\circ}$, respectively. On the other hand, unless major star forming events had occurred in the Galactic disk in the last $100 \mathrm{Myr}$, the present results would favour an important dissolution rate of star clusters.

Acknowledgements. We are gratefully indebted to the CASLEO staff for their hospitality and support during the two observing runs. The authors acknowledge use of the CCD and data acquisition system supported under U.S. National Science Foundation grant AST-90-15827 to R.M. Rich. This work was partially supported by the Argentine institutions CONICET, ANPCyT and CONICOR, the Brazilian institutions $\mathrm{CNPq}$ and FINEP, and the Vitae and Antorchas foundations.

\section{References}

Balona L., Laney C., 1995, MNRAS 277, 250

Batinelli P., Capuzzo-Dolcetta R., 1991, MNRAS 249, 76

Bica E., 1988, A\&A 195, 76

Bica E., Alloin D., 1986a, A\&A 162, 21 (BA86a)

Bica E., Alloin D., 1986b, A\&AS 66, 171 (BA86b)

Bica E., Alloin D., 1987, A\&A 186, 49 (BA87)

Bica E., Alloin D., Santos J.F.C. Jr., 1990, A\&A 235, 103

Bica E., Alloin D., Schmitt H., 1994, A\&A 283, 805

Bica E., Clariá J.J., Bonatto C., Piatti A.E., Ortolani S., Barbuy B., 1995, A\&A 303, 747

Bica E., Clariá J.J., Piatti A.E., Bonatto C., 1998, A\&AS 131, 483

Bonatto C., Bica E., Alloin D., 1995, A\&AS 112, 71

Clariá J.J., Mermilliod J.-C., 1992, A\&AS 95, 429

Clariá J.J., Mermilliod J.-C., Piatti A.E., 1999, A\&AS 134, 301

Collinder P., 1931, Medd. Lunds Astron. Observatorium 2

Crawford D.L., 1978, AJ 83, 48

Feinstein A., Marraco H., 1971, PASP 83, 218

Friel E.D., 1995, ARA\&A 33, 381

Friel E.D., Janes K.A., 1993, A\&A 267, 75

Gutiérrez-Moreno A., Moreno H., Cortés G., Wenderoth E., 1988, PASP 100, 973

Hogg H.R., 1965a, Mem. Mount Stromlo 17

Hogg H.R., 1965b, PASP 77, 440

Janes K.A, Tilley C., Lyngå G., 1988, AJ 95, 771

Kjeldsen H., Frandsen S., 1991, A\&AS 87, 119

Lauberts A., 1982, The ESO/Uppsala Survey of the ESO (B) Atlas, European Southern Observatory, Garching

Lyngå G., 1965, Medd. Lunds Astron. Observatorium S2, N142

Lyngå G., 1987, Catalogue of Open Cluster Data, Strasbourg: Centre de Données Stellaires

Mermilliod J.-C., 1999, WEBDA database, obswww.unige.ch/webda

Meynet G., Mermilliod J.-C., Maeder A., 1993, A\&AS 98, 477

Moffat A.F.J., 1974, A\&A 34, 29

Pacziński B., 1973, in Wolf-Rayet and High-Temperature Stars, IAU Symp. No. 49, Bappu M.K.V. and Sahade J. (eds.). Reidel D. Publ. Co., Dordrecht, p. 143 
Piatti A.E., 1996, Thesis Doctoral, FaMAF, Universidad Nacional de Córdoba, Argentina

Piatti A.E., Bica E., Clariá J.J., 1998a, A\&AS 127, 423

Piatti A.E., Clariá J.J., Abadi M.G., 1995, AJ 110, 2813

Piatti A.E., Clariá J.J., Bica E., Geisler D., Minniti D., 1998b, AJ 116, 801

Piatti A.E., Clariá J.J., Bica E., 1999, MNRAS 303, 65

Pismis P., 1959, Bol. Tonantzintla y Tacubaya 18, 37

Ruprecht J., 1966, Bull. Astron. Czechoslovakia 17, 33

Santos J.F.C. Jr., Bica E., 1993, MNRAS 260, 915

Santos J.F.C. Jr., Bica E., Clariá J.J., Piatti A.E., Girardi L., Dottori H., 1995, MNRAS 276, 1155
Schmidt A., 1988, SPEED User's Manual, Federal University of Santa María, Brazil

Seaton M.J., 1979, MNRAS 187, 73p

Sher D., 1965, MNRAS 129, 237

Smith L.F., Meynet G., Mermilliod J.-C., 1994, A\&A 287, 835

Stone R.P.S., Baldwin J.A., 1983, MNRAS 204, 347

van den Bergh S., Hagen G.L., 1975, AJ 80, 11

Wielen R., 1971, A\&A 13, 309

Wielen R., 1988, in Globular Clusters in Galaxies, Grindlay J.E. and Davis Philip A.G. (eds.). Dordrecht: Reidel, p. 393 